\title{
Effects of the Junction Functionality and Chain Entanglements in Chemomechanical Behavior of Polyelectrolyte Gels
}

\author{
Lianhua Ma, ${ }^{1,2}$ Qingsheng Yang, ${ }^{2}$ and Chunhui Yang ${ }^{3}$ \\ ${ }^{1}$ College of Civil Engineering and Architecture, Hebei University, Baoding 071002, China \\ ${ }^{2}$ Department of Engineering Mechanics, Beijing University of Technology, Beijing 100124, China \\ ${ }^{3}$ School of Computing, Engineering and Mathematics, University of Western Sydney, Penrith, NSW 2751, Australia \\ Correspondence should be addressed to Chunhui Yang; r.yang@uws.edu.au
}

Received 14 October 2014; Revised 30 January 2015; Accepted 8 February 2015

Academic Editor: Yurij Holovatch

Copyright (C) 2015 Lianhua Ma et al. This is an open access article distributed under the Creative Commons Attribution License, which permits unrestricted use, distribution, and reproduction in any medium, provided the original work is properly cited.

\begin{abstract}
By considering the functionality of junctions and entanglements of polymer chains for polyelectrolyte gels, a new free energy density function is presented via combining Edwards-Vilgis slip-link model with Flory-Huggins theory. On this basis, the effects of the functionality of network junctions and entanglements of polymer chains are systematically analyzed for free swelling of a cubic polyelectrolyte gel and constrained swelling of a blanket layer of the gel. Analytical results show that the functionality of junctions and chains entanglements plays an important role in the coupled chemomechanical deformation of polyelectrolyte gels.
\end{abstract}

\section{Introduction}

As a kind of soft active material, the polyelectrolyte gel is constructed by cross-linked polymer network with ionizable groups and interstitial solvent containing anions and cations. Polyelectrolyte gels have an ability to imbibe large amounts of solvents, leading to their wide applications in many fields, such as biomedical devices [1], drug-delivery systems [2], tissue engineering [3], and healthcare situation [4]. Due to the responsiveness to various environmental stimuli, polyelectrolyte gel can be applied as a promising candidate material in sensors and actuators $[5,6]$.

The issue on the chemomechanical behavior of the gel is attracting wide attention. Among the pioneers in studying the polymer gels, Flory and Rehner [7] formulated an explicit free energy density function for gels by taking into account the stretching of the polymer network and the mixing of polymer network and solvent. The classical Flory-Rehner model is still being widely used to study chemomechanical behaviors of gels [8-10]. However, in this model, the free energy of stretching polymer networks is simply described by NeoHookean model where the effects of junction functionality of cross-links and entanglements of polymer chains are not considered. It should be noted that chain entanglements usually exist in nonideal gels because of the uncrossability of polymer chains. The uncross-linked chains are also called slip-links whose entanglements are inevitably produced in preparation of real gels. In view of this issue, Yan and Jin [11] established a hybrid free energy density function for neutral gels by combining Edwards-Vilgis slip-link model [12] of stretching polymer networks with Flory-Huggins theory characterizing the mixing of polymer and solvent. To further examine the effects of the functionality of junctions of the cross-links, Yang et al. [13] constructed a new hybrid free energy density function to investigate the chemomechanical behaviors of polymer gels by several examples. However, their model is restricted to neutral gels. The effects of junction functionality and chain entanglements on the polyelectrolyte gels remain to be resolved.

In the present work, a new hybrid free energy density function is formulated for polyelectrolyte gels through combining Edwards-Vilgis slip-link model [12] of network elasticity with Flory-Huggins model [7] of the network and solution. On this basis, the free swelling and constrained swelling behaviors of the polyelectrolyte gel are analyzed. By comparing with the previous model, the importance of junction functionality and entanglements in polyelectrolyte gels is identified. 


\section{Hybrid Free Energy Function and Constitutive Equations of the Polyelectrolyte Gels}

According to Flory-Huggins polymer solution theory, the free energy of a polyelectrolyte gel originates in three molecular processes: stretching of the polymer network, mixing of the networks and the solvents, and mixing of the solvents and mobile ions. We assume that the ionizable groups on the polymer chains do not dissociate and the fixed charge on the chains is constant. Following prior work [13-16], the total Helmholtz free energy density can be given as

$$
\psi=\psi_{\text {stretch }}+\psi_{\text {mix }}+\psi_{\text {ion }}
$$

where $\psi_{\text {stretch }}$ is the stretching free energy contribution of the polymer networks, $\psi_{\text {mix }}$ is the mixing free energy contribution of the polymer networks and solvents, and $\psi_{\text {ion }}$ is associated with the mixing of solvents and mobile ions. It should be noted that, for the polyelectrolyte gel with dilute solution and weakly charged polymer networks, the ion concentrations of the solution and the density of the fixed charge bound on the polymer networks are relatively low. Therefore, the electrostatic interactions of the ions and fixed charges on the polymer chains are neglected compared to the above-mentioned free energy contribution.

For the elastic free energy of stretching networks, we utilize the Edwards-Vilgis slip-link model to incorporate the effects of chain entanglements. The slippage mechanism of the slip-link is illustrated in Figure 1. Two different chains can be joined with a link through sliding a distance along the contour of chains.

In this model, the energy contribution due to the network stretching can be formulated as [12]

$$
\begin{gathered}
\psi_{\text {stretch }}=\frac{1}{2} N_{c} R T\left\{\sum_{i=1}^{3} \lambda_{i}^{2}-3-\frac{4}{f} \ln J\right. \\
\left.+\frac{N_{s}}{N_{c}} \sum_{i=1}^{3}\left[\frac{(1+\eta) \lambda_{i}^{2}}{1+\eta \lambda_{i}^{2}}+\ln \left(1+\eta \lambda_{i}^{2}\right)\right]\right\},
\end{gathered}
$$

where $N_{c}$ is the mole number of the cross-linked polymer chains; $R$ is the universal gas constant; $T$ is the absolute temperature; $\lambda_{i}(i=1,2,3)$ are the stretches in principal direction; $f$ is the junction functionality of the cross-links; $J$ is the swelling ratio of the gel; $N_{s}$ denotes the mole number of the slip-links; $\eta$ is the slippage parameter.

The free energy of mixing the polymer networks and the solvents can be given as

$$
\psi_{\text {mix }}=\frac{R T}{v}\left(v C_{s} \ln \frac{v C_{s}}{J}+\frac{v C_{s}}{J} \chi\right),
$$

where $v$ and $C_{s}$ are, respectively, the molar volume and mole number of the solvent molecules and $\chi$ is the Flory parameter characterizing the interaction between the solvent and the polymer.

As mentioned above, the concentrations of the ions and the fixed charge bound on the polymer networks are assumed

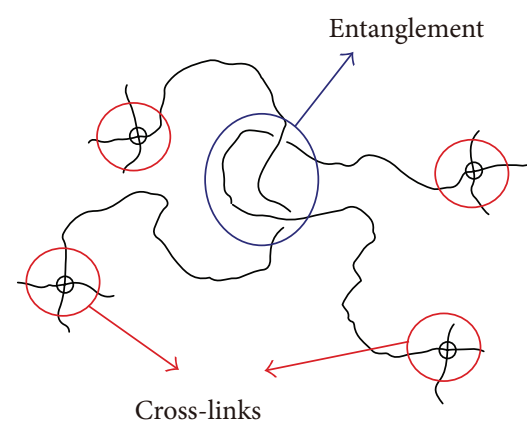

FIGURE 1: Schematics of a typical structure of a polymer network with entanglements.

to be relatively low, and the ion electrostatic interactions in the gel are generally ignored [14]. In this situation, $\psi_{\text {ion }}$ is dominated by the mixing entropy of the solvents and ionic species [16]. Consider

$$
\psi_{\text {ion }}=\psi_{i}^{\mathrm{ref}}+\mu_{\beta}^{\mathrm{ref}} C_{\beta}+R T \sum_{\beta=s,+,-} C_{\beta}\left(\ln \frac{C_{\beta}}{\sum_{\beta=s,+,-} C_{\beta}}\right) \text {, }
$$

where $\psi_{i}^{\text {ref }}$ is the reference free energy of mobile ions; $\mu_{\beta}^{\text {ref }}$ and $C_{\beta}$ denote the reference chemical potential and mole number of the species $\beta(\beta=s,+,-)$, where the subscripts $s,+$, and denote the solvent, cations, and anions, respectively.

According to Hong et al. [8], we introduce an assumption that the polymer chains and species component are individually incompressible. Furthermore, in the case of polyelectrolyte gels, the concentrations of ions are assumed to be much lower than those of the solvents [15]. As a condensed matter, the gel has negligible void space, and its volume can be given as the sum of the volume of the dry polymer network and that of the solvents; that is,

$$
J=1+v C_{s}
$$

To take the solvent chemical potential as the independent variable, we naturally introduce another free energy density function by using a Legendre transformation:

$$
\widehat{\psi}=\psi-\mu C_{s} .
$$

For the general deformations under complex mechanical loadings, (6) can be written in terms of principal invariants $I_{i}(i=1,2,3)$. In the framework of nonlinear continuum mechanics, let $\mathbf{F}$ be the deformation gradient tensor of a body subjected to mechanical loading, $\mathbf{C}=\mathbf{F}^{T} \mathbf{F}$; then the first, second, and third principal invariants of $\mathbf{C}$ are expressed, respectively, as

$$
\begin{aligned}
& I_{1}=\operatorname{tr} \mathbf{C}, \\
& I_{2}=\operatorname{tr} \mathbf{C}^{2}, \\
& I_{3}=\operatorname{det} \mathbf{C}=J^{2} .
\end{aligned}
$$


Substituting (2)-(4) into (1) and (6) and using (5) and (7), we obtain the free energy function $\widehat{\psi}$ as follows:

$$
\begin{aligned}
\widehat{\psi}(\mathbf{F}, \mu) & =\frac{1}{2} N_{c} R T\left\{I_{1}-3-\frac{4}{f} \ln J+\frac{N_{s}}{N_{c}}\right. \\
\cdot & {\left.\left[\frac{(1+\eta)\left(I_{1}+2 \eta I_{2}+3 \eta^{2} J^{2}\right)}{1+\eta I_{1}+\eta^{2} I_{2}+\eta^{3} J^{2}}+\ln \left(1+\eta I_{1}+\eta^{2} I_{2}+\eta^{3} J^{2}\right)\right]\right\} } \\
- & \frac{R T}{v}\left[(J-1) \ln \left(\frac{J}{J-1}\right)-\frac{J-1}{J} \chi\right]+R T \sum_{\gamma=+,-} C_{\gamma} \ln \frac{v C_{\gamma}}{J-1} \\
& -\frac{\mu}{v}(J-1) .
\end{aligned}
$$

It is readily found that function (8) is singular when the gel is in dry state; that is, $J=1$. To overcome this, we take the swollen state of the gel as a reference state, where the initial equilibrium between the mechanical stretching and chemical mixing is reached without external mechanical loads and constraints. Relative to the dry state, the gel in this state freely swells with a stretch $\lambda_{0}$; therefore the initial deformation gradient $\mathbf{F}_{0}$ in the referenced state can be expressed as

$$
\mathbf{F}_{0}=\operatorname{diag}\left[\begin{array}{lll}
\lambda_{0} & \lambda_{0} & \lambda_{0}
\end{array}\right]
$$

Choosing the initially swollen state as a reference state, the deformation gradient $\mathbf{F}$ from the dry state to the current state is

$$
\mathbf{F}=\mathbf{F}^{\prime} \mathbf{F}_{0}
$$

where $\mathbf{F}^{\prime}$ is the deformation gradient from the initially swollen state to the current state. Correspondingly, the right Cauchy-Green tensor can be given as $\mathbf{C}^{\prime}=\mathbf{F}^{\prime T} \mathbf{F}^{\prime}$.

Recalling the definition of free energy density in the reference state, we have the following relationship:

$$
\widehat{\psi}^{\prime}\left(\mathbf{F}^{\prime}, \mu\right)=\lambda_{0}^{-3} \widehat{\psi}(\mathbf{F}, \mu)
$$

Substituting (8) and (10) into (11), we obtain

$$
\begin{aligned}
& \widehat{\psi}^{\prime}\left(\mathbf{F}^{\prime}, \mu\right)=\frac{1}{2} N_{c} R T\left[\lambda_{0}^{-1} I_{1}^{\prime}-\frac{3}{\lambda_{0}^{3}}\right. \\
& \left.-\frac{4}{f \lambda_{0}^{3}}\left(3 \ln \lambda_{0}+\ln J^{\prime}\right)\right]+\frac{\lambda_{0}^{-3}}{2} \\
& \cdot N_{s} R T\left[\frac{(1+\eta)\left(\lambda_{0}^{2} I_{1}^{\prime}+2 \lambda_{0}^{4} \eta I_{2}^{\prime}+3 \lambda_{0}^{6} \eta^{2} J^{\prime 2}\right)}{1+\lambda_{0}^{2} \eta I_{1}^{\prime}+\lambda_{0}^{4} \eta^{2} I_{2}^{\prime}+\lambda_{0}^{6} \eta^{3} J^{\prime 2}}\right. \\
& \left.+\ln \left(1+\lambda_{0}^{2} \eta I_{1}^{\prime}+\lambda_{0}^{4} \eta^{2} I_{2}^{\prime}+\lambda_{0}^{6} \eta^{3} J^{\prime 2}\right)\right] \\
& -\frac{R T}{v_{s}}\left[\left(J^{\prime}-\lambda_{0}^{-3}\right) \ln \left(\frac{J^{\prime}}{J^{\prime}-\lambda_{0}^{-3}}\right)-\frac{\chi\left(J^{\prime}-\lambda_{0}^{-3}\right)}{\lambda_{0}^{3} J^{\prime}}\right] \\
& +R T \lambda_{0}^{-3} \sum_{\gamma=+,-} C_{\gamma} \ln \frac{v C_{\gamma}}{\lambda_{0}^{3} J^{\prime}-1}-\frac{\mu}{v}\left(J^{\prime}-\lambda_{0}^{-3}\right),
\end{aligned}
$$

where $I_{1}^{\prime}=\operatorname{tr} \mathbf{C}^{\prime}, I_{2}^{\prime}=\operatorname{tr} \mathbf{C}^{\prime 2}$, and $J^{\prime}=\operatorname{det} \mathbf{F}^{\prime}$. As observed in (12), the stretching part of the free energy relates to the junction functionality of the cross-links. For a polymer network with any connectivity, the functionality generally satisfies $f>2$. It is emphasized that the junction functionality and the entanglements of chains were neglected in many previous studies [7-10].

It should be pointed out that, at the initially swollen state of the polyelectrolyte gel, the nominal stress vanishes. Accordingly, the derivative of (8) with respect to $J$ equals zero, leading to the relationship between initial stretch $\lambda_{0}$ and the initial chemical potential $\mu_{0}$ :

$$
\begin{aligned}
& N_{c}\left(\lambda_{0}^{-1}-\frac{2 \lambda_{0}^{-3}}{f}\right)+\frac{1}{v}\left(\ln \left(1-\lambda_{0}^{-3}\right)+\lambda_{0}^{-3}+\lambda_{0}^{-6} \chi\right) \\
& +N_{s}(1+\eta)\left[\frac{\lambda_{0}^{-1}+4 \eta \lambda_{0}+3 \eta^{2} \lambda_{0}^{3}}{1+3 \eta \lambda_{0}^{2}+3 \eta^{2} \lambda_{0}^{4}+\eta^{3} \lambda_{0}^{6}}\right. \\
& +\frac{\eta \lambda_{0}^{-1}+2 \eta^{2} \lambda_{0}+\eta^{3} \lambda_{0}^{3}}{(1+\eta)\left(1+3 \eta \lambda_{0}^{2}+3 \eta^{2} \lambda_{0}^{4}+\eta^{3} \lambda_{0}^{6}\right)} \\
& \left.-\frac{\left(3 \lambda_{0}^{2}+6 \eta \lambda_{0}^{4}+3 \eta^{2} \lambda_{0}^{6}\right)\left(\eta \lambda_{0}^{-1}+2 \eta^{2} \lambda_{0}+\eta^{3} \lambda_{0}^{3}\right)}{\left(1+3 \eta \lambda_{0}^{2}+3 \eta^{2} \lambda_{0}^{4}+\eta^{3} \lambda_{0}^{6}\right)^{2}}\right] \\
& =\sum_{\gamma=+,-} \frac{C_{\gamma}}{\lambda_{0}^{3}-1}+\frac{\mu_{0}}{R T v} .
\end{aligned}
$$

For the external solution which is supposed to be ideal dilute solution, the chemical potential $\mu_{0}$ of the solvent is given by [13]

$$
\mu_{0} \approx-R T v \sum_{\gamma=+,-} c_{\gamma}^{e}
$$

where $c_{\gamma}^{e}(\gamma=+,-)$ denotes the concentration of mobile ion $\gamma$ in the surrounding solution.

For simplicity, we assume that the mobile ions are monovalent. Moreover, the condition of electroneutrality is supposed to be satisfied both in the polyelectrolyte gel and in the surrounding solution; therefore

$$
\begin{aligned}
c_{+}-c_{-}+z^{\mathrm{fc}} c^{\mathrm{fc}} & =0, \\
c_{+}^{e} & =c_{-}^{e}=\bar{c},
\end{aligned}
$$

where $c_{\gamma}(\gamma=+,-)$ is the concentration of ion $\gamma$ per unit solution volume inside the polyelectrolyte gel and $z^{\mathrm{fc}}$ and $c^{\mathrm{fc}}$ are the valence and the fixed charge density on the chains, respectively. In the equilibrium state, the chemical potential of mobile ions inside and outside the gel is identical. Recalling the conditions of mass conservation and electroneutrality, we have

$$
\sum_{\gamma=+,-} \frac{C_{\gamma}}{\lambda_{0}^{3}-1}=\sqrt{\frac{\left(z^{\mathrm{fc}} C^{\mathrm{fc}}\right)^{2}}{\left(\lambda_{0}^{3}-1\right)^{2}}+4 \bar{c}^{2}} .
$$

It is important to point out that $C_{\gamma}$ and $C^{\mathrm{fc}}$ are the nominal concentration of the internal mobile ion $\gamma$ and the nominal density of the fixed charges, respectively. 


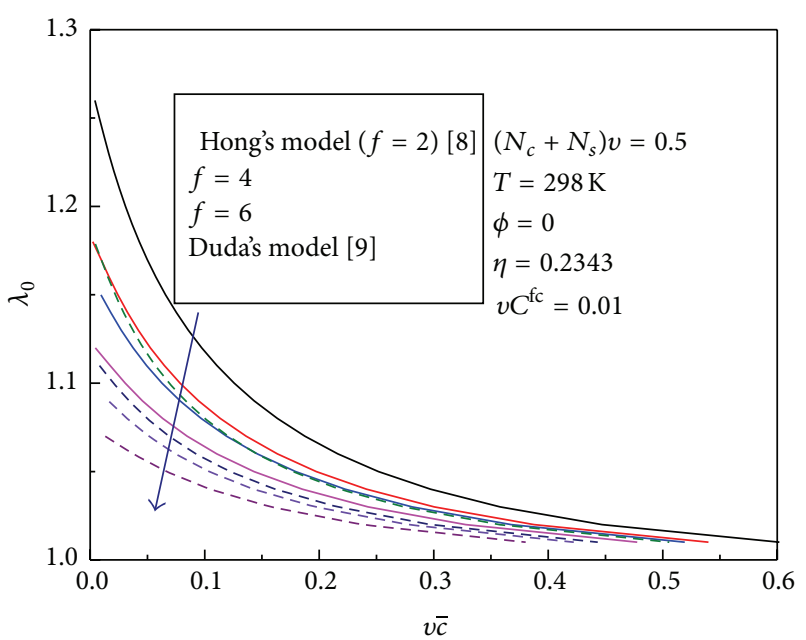

(a1)

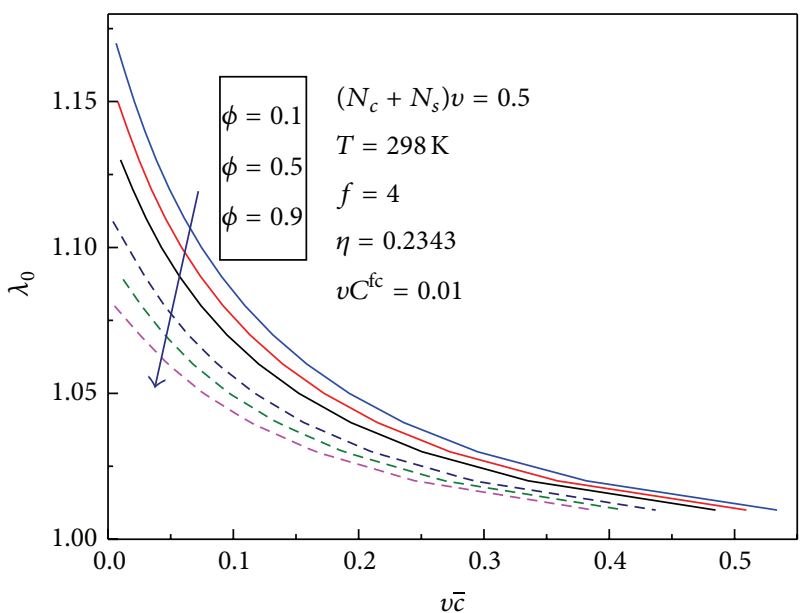

(b1)

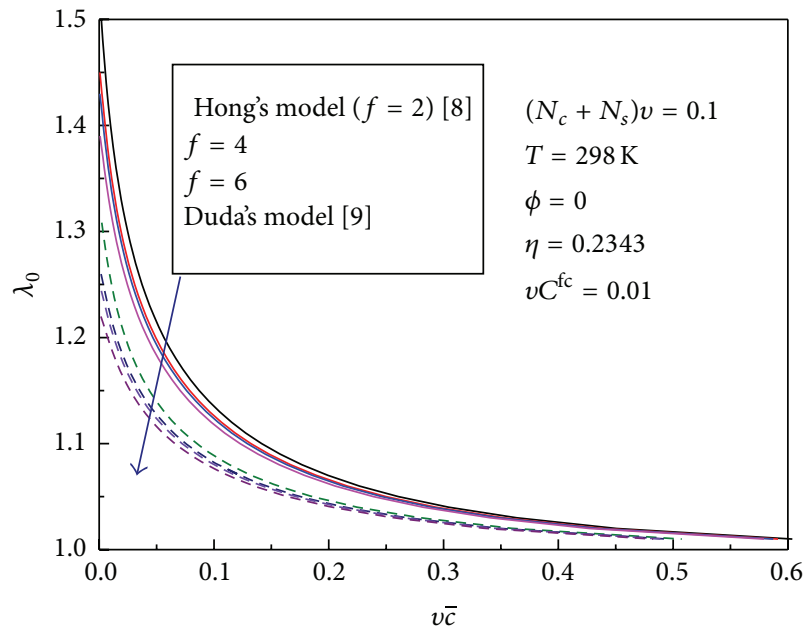

(a2)

(a)

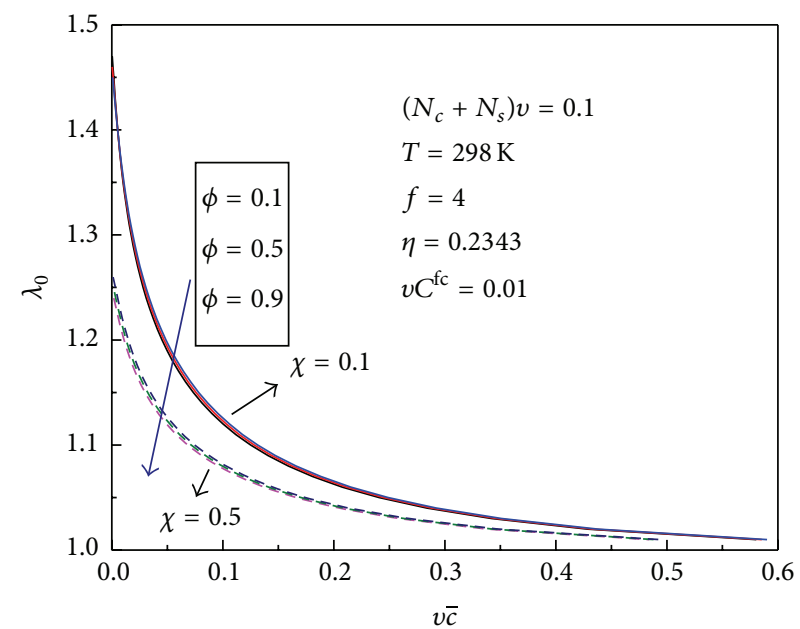

(b2)

(b)
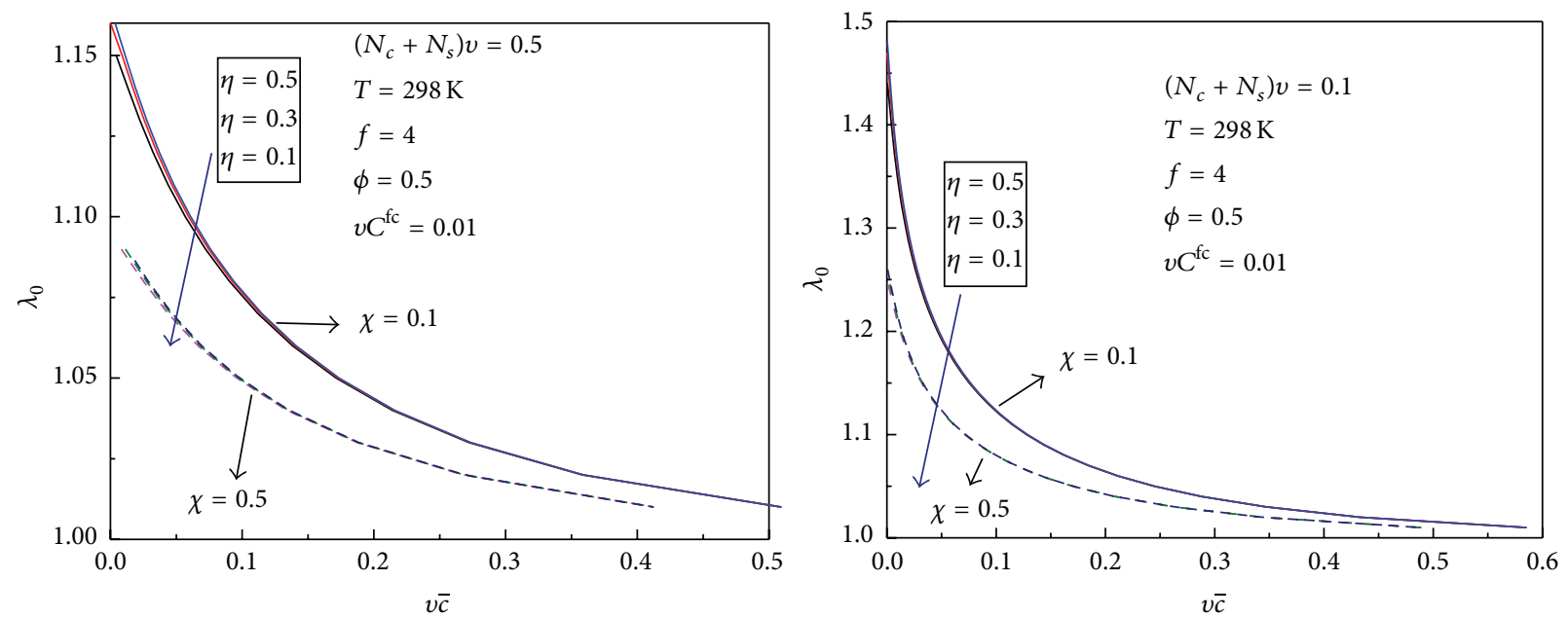

$\begin{aligned} \chi & =0.1 \\ --\chi & =0.5\end{aligned}$

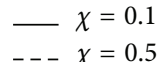

(c1)

(c)

Figure 2: Continued. 


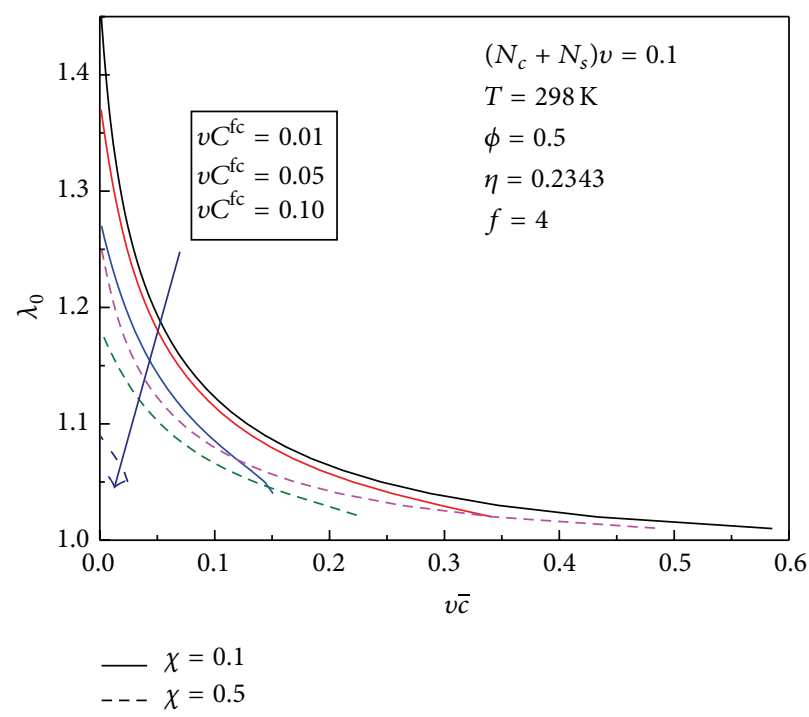

(d)

Figure 2: Variations of the stretch of a cubic polyelectrolyte gel with the ion concentrations of the external solution for different microstructural parameters.

It is readily observed that the initially swollen polyelectrolyte gel can be viewed as a hyperelastic material. In the framework of nonlinear continuum mechanics, we introduce the first Piola-Kirchhoff stress tensor, $\mathbf{S}$, which is expressed as

$$
\mathbf{S}=\frac{\partial \widehat{\psi}^{\prime}}{\partial \mathbf{F}^{\prime}}
$$

Neglecting the body force, the mechanical governing equation in equilibrium state satisfies

$$
\operatorname{Div} \mathbf{S}=0 \text {, }
$$

where Div represents the divergence operator. Let $\sigma$ denote the Cauchy stress tensor; then $\boldsymbol{\sigma}$ and $\mathbf{S}$ are connected by $\boldsymbol{\sigma}=$ $J^{-1} \mathbf{S F}^{T}$.

\section{Analytical Results}

In this section, two examples of homogeneous deformations of polyelectrolyte gel are analyzed by utilizing the developed hybrid free energy density function, and the effects of microstructural parameters on the chemomechanical behavior of the gel are addressed. For convenience, the molar concentrations of mobile ions in the surrounding solution are normalized by $1 / v$, and the stress is normalized by $R T / v$. Several dimensionless parameters, $N_{c} v, N_{s} v, f, \eta, \chi$, and $v C^{\mathrm{fc}}$, are involved in the proposed free energy density function. For the polyelectrolyte gels, $\left(N_{c}+N_{c}\right) v$ generally has the range of $10^{-4}-10^{-1}$, and the values of $\eta$ and $\chi$ are both taken in the allowable range of $0.1-0.5$.

3.1. Free Swelling of a Cubic Polyelectrolyte Gel. When immersed in an external solution, a cubic polyelectrolyte gel subject to no external mechanical loads and constraints may swell freely until the equilibrium of the migrations of solvents

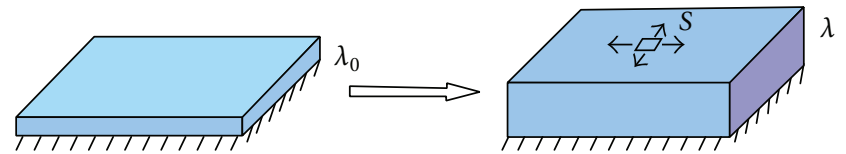

FIGURE 3: Schematics of the constrained swelling of a blanket gel layer attached to a rigid substrate.

and mobile ions is achieved. At this point, the chemical potential of the interior solvent is identical to that of the exterior solvent. While the ion concentrations of the external solution experience changes, the polyelectrolyte gel can swell or shrink due to the migrations of mobile species. In these circumstances, the stretch $\lambda_{0}$ of the gel can be related to the ion concentration $\bar{c}$ of the external solution by (13) and (14). We define a parameter $\phi=N_{s} /\left(N_{c}+N_{s}\right)$ to characterize the volume ratio of the slip-links in the total networks of the polyelectrolyte gel. Through a systematic calculation for different material parameters $f, \phi, \eta$, and $C^{\mathrm{fc}}$, the elongation $\lambda_{0}$ of the cubic gel with respect to the dry state is plotted against the ion concentrations of the external solution in Figures 2(a)-2(d).

It can be found from Figure 2 that the elongation $\lambda_{0}$ of the polyelectrolyte gel is directly dependent on the external ion concentration. When the ion concentration decreases, the swelling ratio of the gel increases in a nonlinear manner. Moreover, the microstructural parameters have important effects on the swelling deformation of the polyelectrolyte gel. For instance, in the dilute case of the external solution where the ion concentration is relatively small, the decreasing junction functionality $f$ induces the increasing swelling ratio of the gel. This variation trend is relatively strong for the case of high concentration of polymer links. The results from Hong et al. [8] and Duda et al. [9] are also plotted for comparison. It can be found in Figures 2(a1)-2(a2), for the general polyelectrolyte gel, that the results are overestimated by 


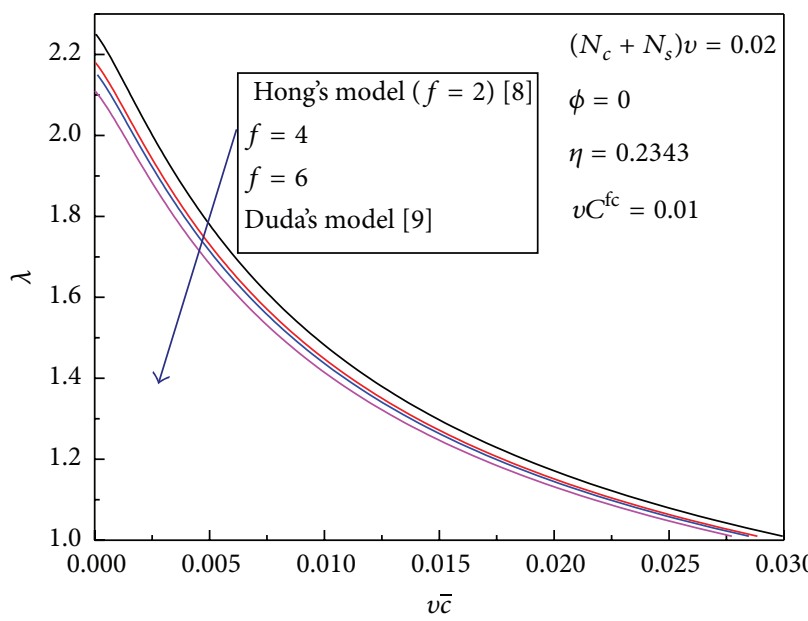

(a1)

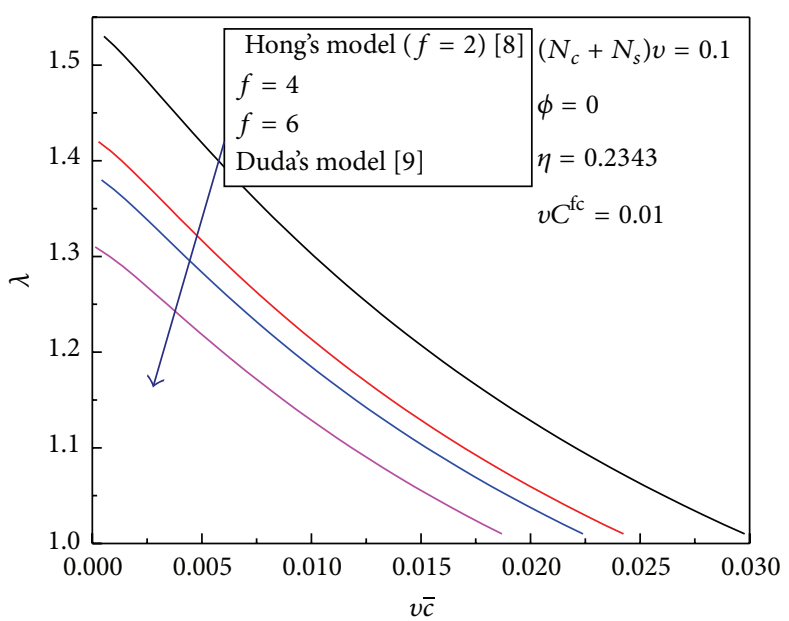

(a2)

(a)

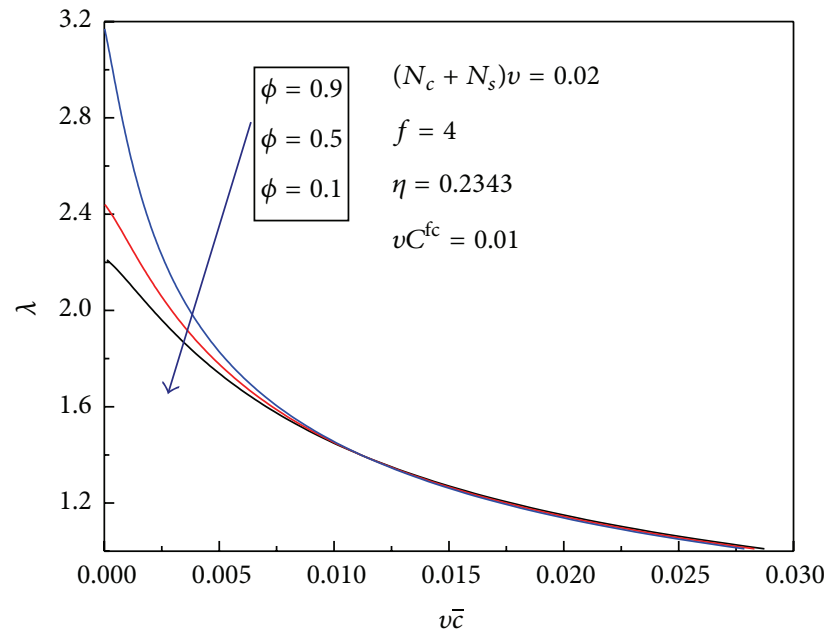

(b1)

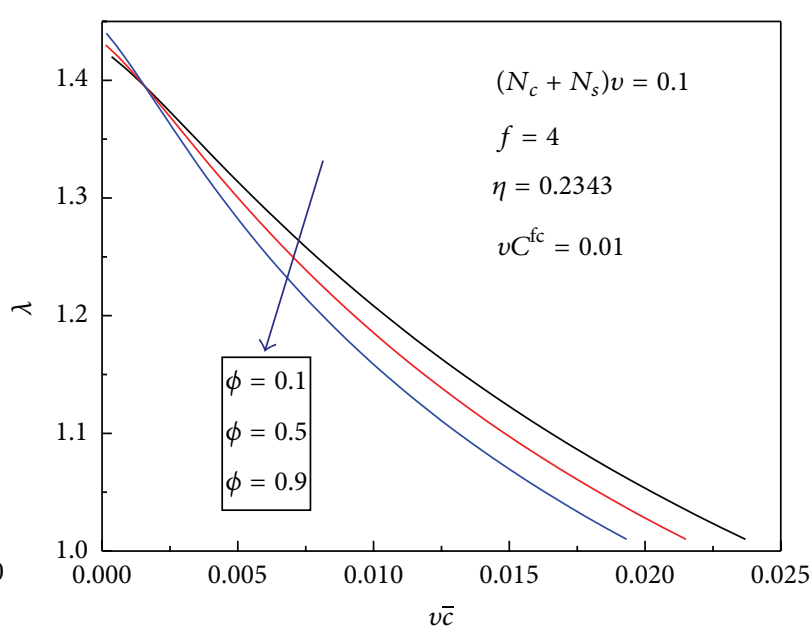

(b2)

(b)

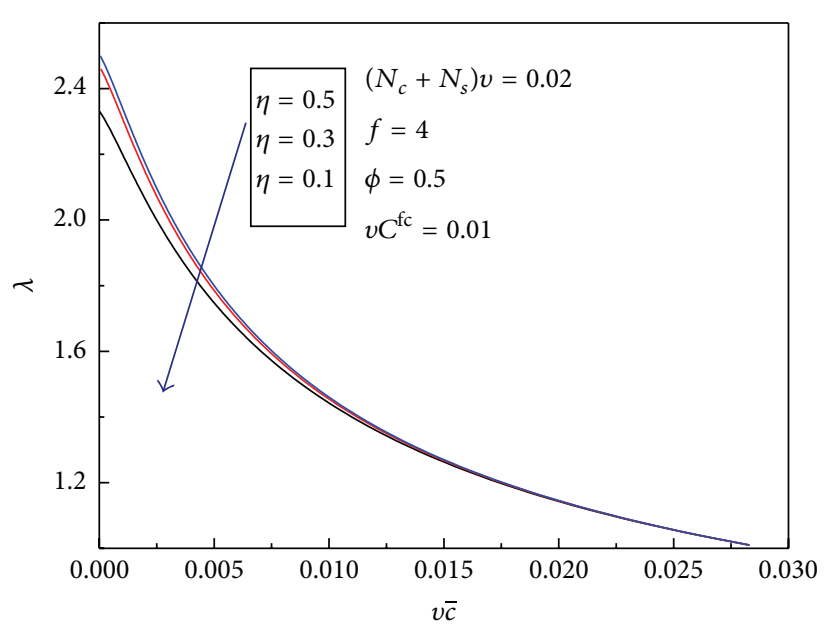

(c1)

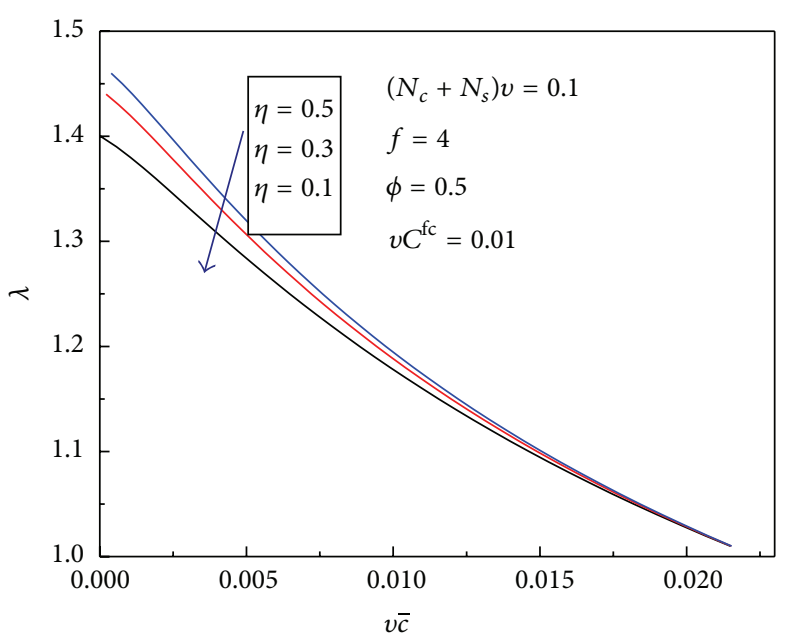

(c2)

(c)

Figure 4: Continued. 


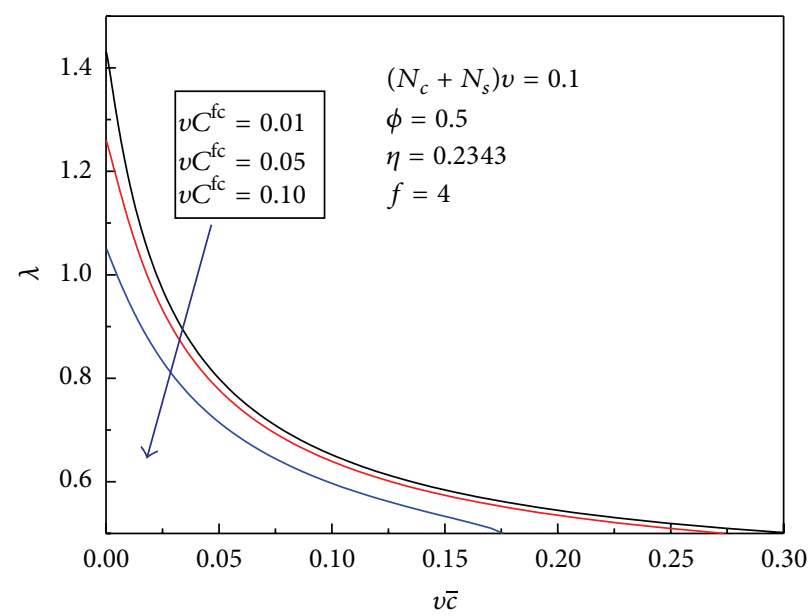

(d)

FIGURE 4: Variations of the stretch normal to the blanket layer of a polyelectrolyte gel attached to a rigid substrate with the external ion concentrations for different microstructural parameters.

Hong's model and underestimated by Duda's model. It is needed to note that the effects of junction functionality of polyelectrolyte gels on free swelling are similar to those of neutral gels [13]. For the effects of material parameters $\phi$ and $\eta$, when the external ion concentration holds to be dilute, the higher value of $\phi$ corresponds to the lower stretch, but for $\eta$, the opposite is true. While the external ion concentration is relatively large, the gel swells less and the effects of $f, \phi$, and $\eta$ are negligible. It can be also observed that the high fixed charge density suppresses the swelling of the polyelectrolyte gel. Generally, when the solution is very dilute, the induced swelling deformation of the polyelectrolyte gel is large, and the effects of the microstructural parameters are not neglected, especially for the case of high concentration of polymer links.

\subsection{Constrained Swelling of a Blanket Layer of Polyelectrolyte}

Gel. Following Hong et al. [17] and Yang et al. [13], a layer of a polyelectrolyte gel tied to a rigid substrate is further considered, and the initial stretch of the gel is assumed to be $\lambda_{0}=1.5$. Then subsequently, the gel is immersed in a solution with different ion concentrations. Due to the constraint of the substrate, the gel layer swells or shrinks to a stretch $\lambda$ along the normal direction of the blanket gel layer and achieves another equilibrium state with equal-biaxial stress, as shown in Figure 3. have

The stress normal to the blanket layer vanishes, and we

$$
\begin{gathered}
\frac{N_{c} v}{\lambda_{0}^{2}}\left(\lambda-\frac{2}{f \lambda}\right)+\frac{N_{s} v}{\lambda_{0}^{2}}\left[\frac{(1+\eta) \lambda}{\left(1+\eta \lambda^{2}\right)^{2}}+\frac{\eta \lambda}{1+\eta \lambda^{2}}\right] \\
+\ln \left(1-\frac{1}{\lambda \lambda_{0}^{2}}\right)+\frac{1}{\lambda \lambda_{0}^{2}}+\frac{\chi}{\left(\lambda \lambda_{0}^{2}\right)^{2}}+2 v \bar{c} \\
\quad-\sqrt{\frac{\left(z^{\mathrm{fc}} v C^{\mathrm{fc}}\right)^{2}}{\left(\lambda \lambda_{0}^{2}-1\right)^{2}}+4 v^{2} \bar{c}^{2}}=0,
\end{gathered}
$$

where the two in-plane stretches hold to be the initial value $\lambda_{0}$ due to the confinement by the rigid substrate, whereas the out-of-plane stretch can undergo variations with the external ion concentrations of the solution. The normalized in-plane biaxial stress can be expressed as

$$
\begin{aligned}
& \frac{v S}{R T}=N_{c} v\left(\lambda_{0}-\frac{2}{f \lambda_{0}}\right)+N_{s} v\left[\frac{(1+\eta) \lambda_{0}}{\left(1+\eta \lambda_{0}^{2}\right)^{2}}\right. \\
& \left.+\frac{\eta \lambda_{0}}{1+\eta \lambda_{0}^{2}}\right]+\frac{1}{\lambda_{0}}\left[\lambda \lambda_{0}^{2} \ln \left(1-\frac{1}{\lambda \lambda_{0}^{2}}\right)+1+\frac{\chi}{\lambda \lambda_{0}^{2}}\right. \\
& +2 \lambda \lambda_{0}^{2} v \bar{c}-\lambda \lambda_{0}^{2} \sqrt{\left.\frac{\left(z^{\mathrm{fc}} v C^{\mathrm{fc}}\right)^{2}}{\left(\lambda \lambda_{0}^{2}-1\right)^{2}}+4 v^{2} \bar{c}^{2}\right] .}
\end{aligned}
$$

With the use of (19), we plot the stretch $\lambda$ normal to the blanket layer against the ion concentrations of the external solution for different values of microstructural parameters, as shown in Figure 4. Similar to the free swelling, the higher value of the functionality $f$ causes the blanket gel layer to swell less in the normal direction of the layer, and the effect is significant especially for the case of high concentration of the polymer links. It is interesting to note that the effect of the volume ratio of slip-links $\phi$ depends on the concentration of polymer links. When the value of $\left(N_{c}+N_{s}\right) v$ is relatively small, the high value of $\phi$ can induce the large normal stretch of the gel layer in the case of dilute solution. However, when the concentration of polymer links is sufficiently high, a critical value of $v \bar{c}$ can be found as shown in Figure 4(b2). When the external ion concentration is lower than the critical value, the normal stretch of the gel layer increases with the increase of the value of $\phi$, but when the external ion concentration is higher than the critical value, the normal stretch decreases with the increase of the value of $\phi$. The effects of the parameter 


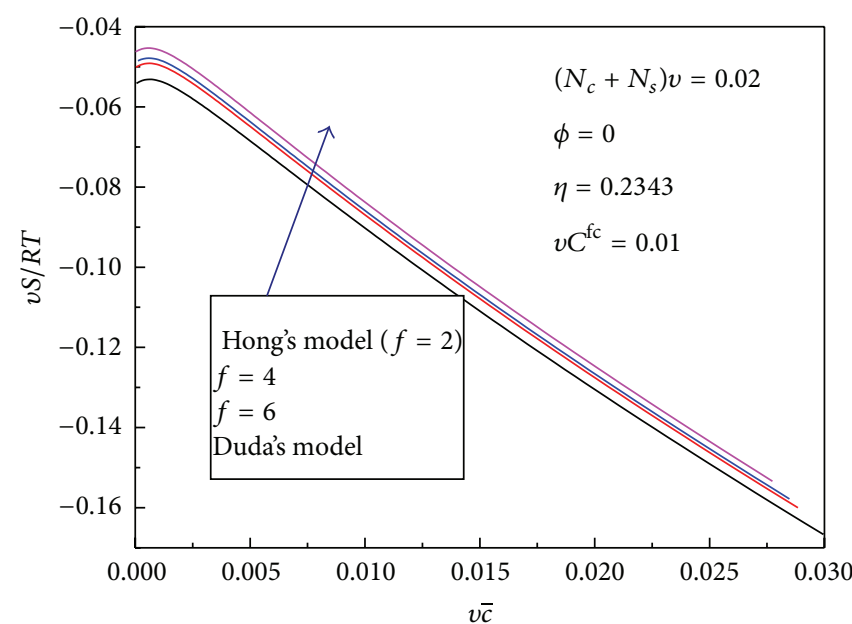

(a1)

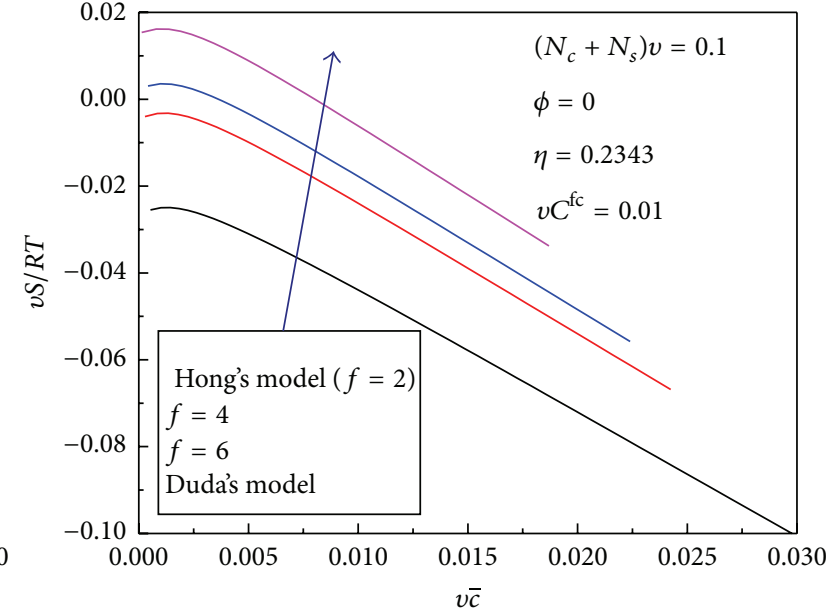

(a2)

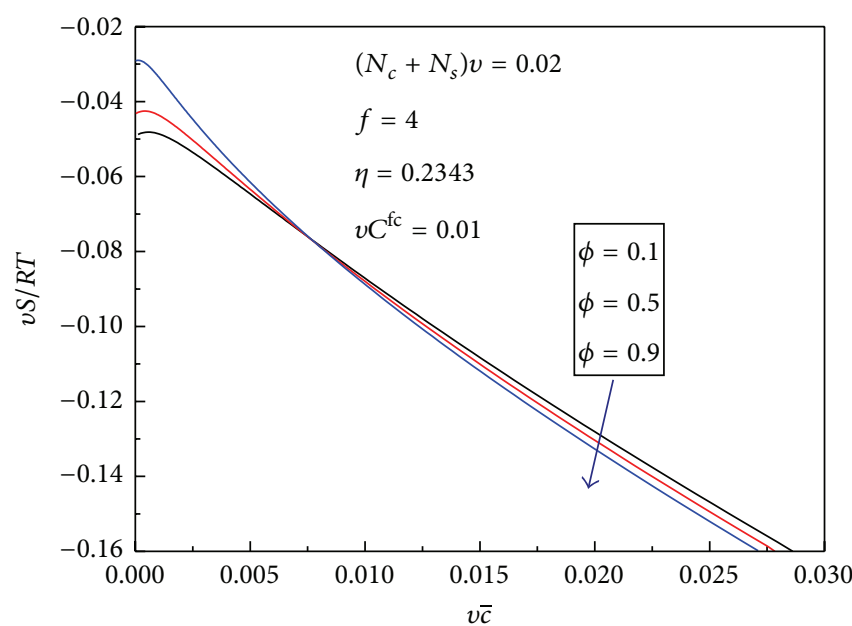

(b1)

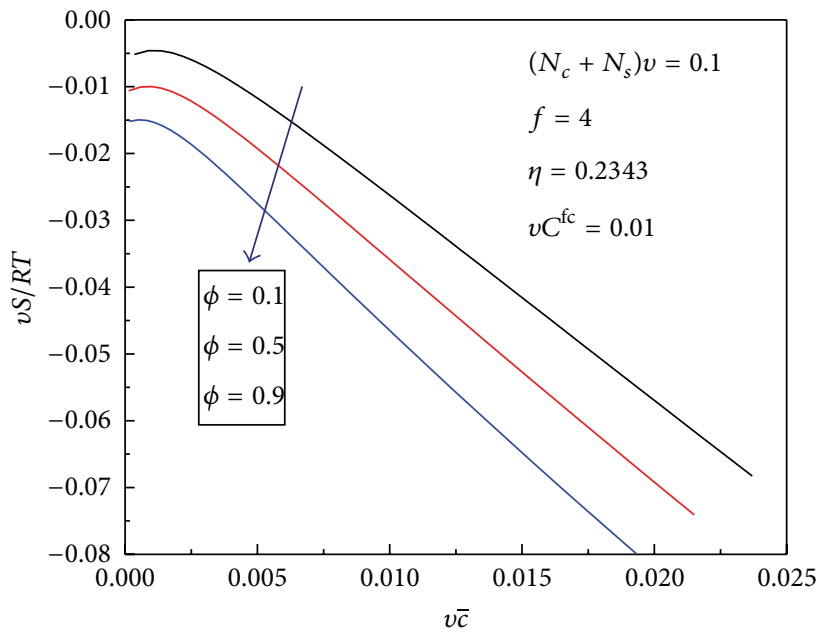

(b2)

(b)

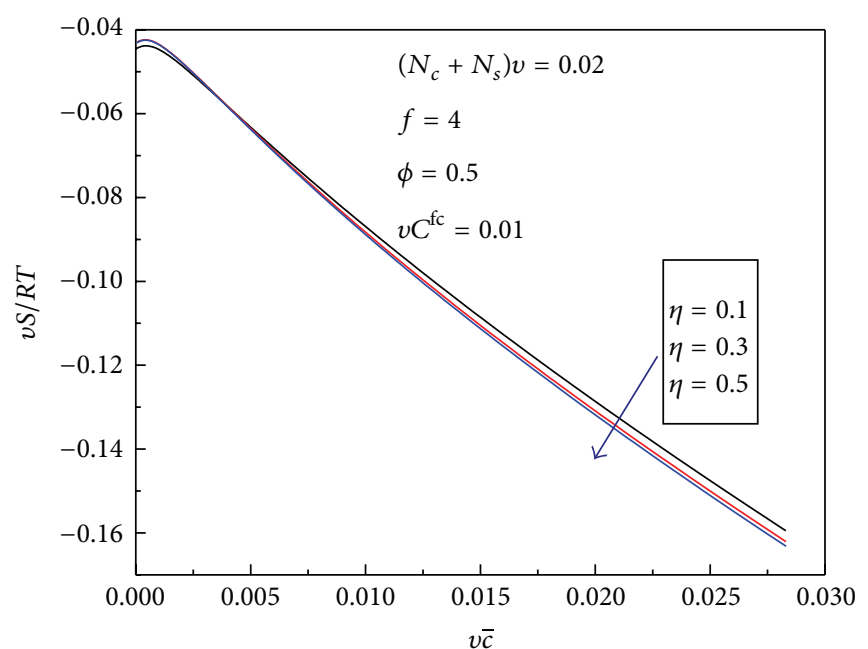

(c1)

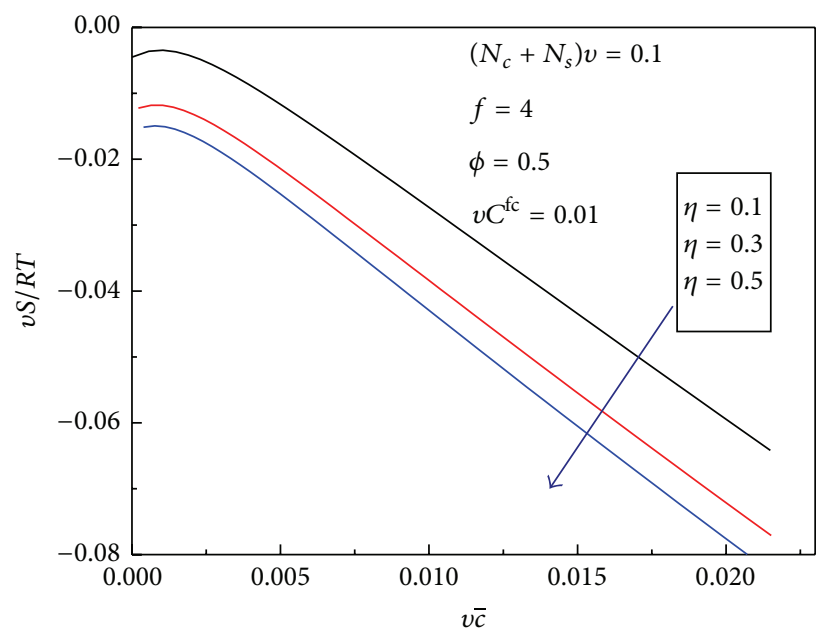

(c2)

(c)

Figure 5: Continued. 


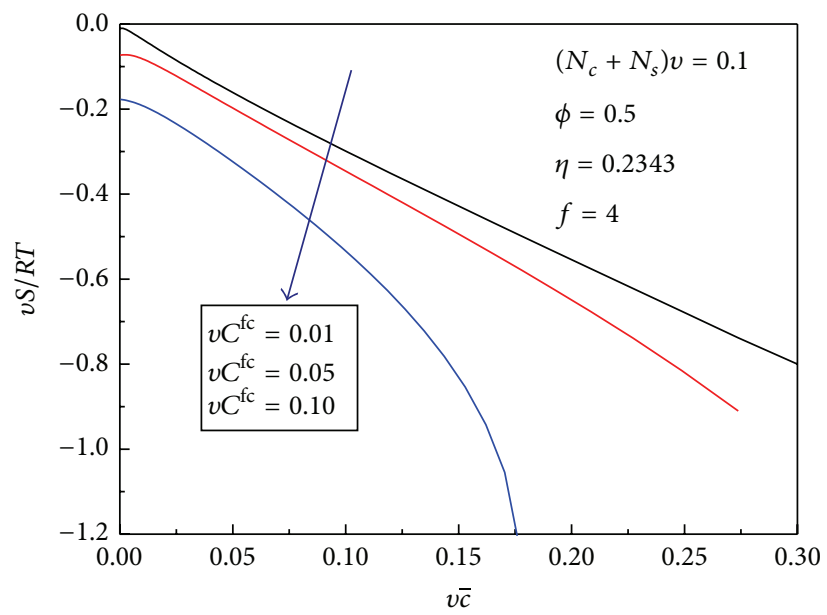

(d)

FiguRE 5: Variations of the produced equal-biaxial stress of the blanket layer of a polyelectrolyte gel with the external ion concentrations for different microstructural parameters.

$\eta$ are similar to the free swelling case. The gel layer swells less along the normal direction when $\eta$ is lower, and the effect is quite significant for the case of the external dilute solution and the high concentration of the polymer links, as shown in Figure 4(c2). Similar to the case of free swelling, Figure 4(d) shows that the higher fixed charge density induces the lower normal stretch of the blanket layer of a polyelectrolyte gel.

Upon chemomechanical deformation, an in-plane compressive stress is induced in the blanket layer of the polyelectrolyte gel due to the constraint of the rigid substrate. According to (20), the normalized equal-biaxial compressive stress curve against the ion concentrations of the external solution for different values of microstructural parameters is plotted as shown in Figure 5. It can be readily observed that the micromechanical parameters have significant impact on the produced equal-biaxial stress, especially when the gel has the large concentration of polymer links.

\section{Conclusions}

Considering the junction functionality and the chain entanglement, a new hybrid free energy density function for polyelectrolyte gels has been formulated with neglecting the electrostatic interactions of the ions and fixed charges and the chemomechanical equilibrium model has been established. The analytical method has been employed to investigate the effects of microstructural parameters on the chemomechanical behaviors of the polyelectrolyte gels. Analytical results show that the junction functionality and the chain entanglements have important effects on the chemomechanical behavior of the polyelectrolyte gels with large concentration of polymer chains. The proposed free energy density function and the corresponding material model will be implemented in the finite element analysis package Abaqus using a userdefined subroutine as a part of future work and will provide a useful tool to model diverse phenomena in polyelectrolyte gels, such as inhomogeneous large deformation, contact, and swell-induced surface instability.

\section{Conflict of Interests}

The authors declare that there is no conflict of interests regarding the publication of this paper.

\section{Acknowledgments}

The financial support from the Natural Science Foundation of China under Grant nos. 11172012 and 11472020 and the Municipal Natural Science Foundation of Beijing under Grant no. 3092006 is gratefully acknowledged.

\section{References}

[1] N. A. Peppas, J. Z. Hilt, A. Khademhosseini, and R. Langer, "Hydrogels in biology and medicine: from molecular principles to bionanotechnology," Advanced Materials, vol. 18, no. 11, pp. 1345-1360, 2006.

[2] Y. Qiu and K. Park, "Environment-sensitive hydrogels for drug delivery," Advanced Drug Delivery Reviews, vol. 53, no. 3, pp. 321-339, 2001.

[3] K. T. Nguyen and J. L. West, "Photopolymerizable hydrogels for tissue engineering applications," Biomaterials, vol. 23, no. 22, pp. 4307-4314, 2002.

[4] S. A. Dubrovskii, M. V. Afanas'eva, M. A. Lagutina, and K. S. Kazanskii, "Comprehensive characterization of superabsorbent polymer hydrogels," Polymer Bulletin, vol. 24, no. 1, pp. 107-113, 1990.

[5] T. Tanaka, D. Fillmore, S.-T. Sun, I. Nishio, G. Swislow, and A. Shah, "Phase transitions in ionic gels," Physical Review Letters, vol. 45 , no. 20, pp. 1636-1639, 1980.

[6] I. Ohmine, "Salt effects on the phase transition of ionic gels," The Journal of Chemical Physics, vol. 77, no. 11, pp. 5725-5729, 1982.

[7] P. J. Flory and J. Rehner, "Statistical mechanics of cross-linked polymer networks.II. Swelling," The Journal of Chemical Physics, vol. 11, no. 11, pp. 521-526, 1943.

[8] W. Hong, X. H. Zhao, J. Zhou, and Z. G. Suo, "A theory of coupled diffusion and large deformation in polymeric gels," 
Journal of the Mechanics and Physics of Solids, vol. 56, no. 5, pp. 1779-1793, 2008.

[9] F. P. Duda, A. C. Souza, and E. Fried, "A theory for species migration in a finitely strained solid with application to polymer network swelling," Journal of the Mechanics and Physics of Solids, vol. 58, no. 4, pp. 515-529, 2010.

[10] S. Baek and T. J. Pence, "Inhomogeneous deformation of elastomer gels in equilibrium under saturated and unsaturated conditions," Journal of the Mechanics and Physics of Solids, vol. 59, no. 3, pp. 561-582, 2011.

[11] H. X. Yan and B. Jin, "Influence of microstructural parameters on mechanical behavior of polymer gels," International Journal of Solids and Structures, vol. 49, no. 3-4, pp. 436-444, 2012.

[12] S. F. Edwards and T. A. Vilgis, "The effect of entanglements in rubber elasticity," Polymer, vol. 27, no. 4, pp. 483-492, 1986.

[13] Q. S. Yang, L. H. Ma, and J. J. Shang, “The chemo-mechanical coupling behavior of hydrogels incorporating entanglements of polymer chains," International Journal of Solids and Structures, vol. 50, no. 14-15, pp. 2437-2448, 2013.

[14] J. Rička and T. Tanaka, "Swelling of ionic gels: quantitative performance of the Donnan theory," Macromolecules, vol. 17, no. 12, pp. 2916-2921, 1984.

[15] R. Marcombe, S. Cai, W. Hong, X. Zhao, Y. Lapusta, and Z. Suo, "A theory of constrained swelling of a $\mathrm{pH}$-sensitive hydrogel," Soft Matter, vol. 6, no. 4, pp. 784-793, 2010.

[16] L. H. Ma, Micromechanics and multiphysics of porous materials saturated with fluid [Ph.D. dissertation], Beijing University of Technology, Beijing, China, 2012.

[17] W. Hong, Z. Liu, and Z. Suo, "Inhomogeneous swelling of a gel in equilibrium with a solvent and mechanical load," International Journal of Solids and Structures, vol. 46, no. 17, pp. 3282-3289, 2009. 

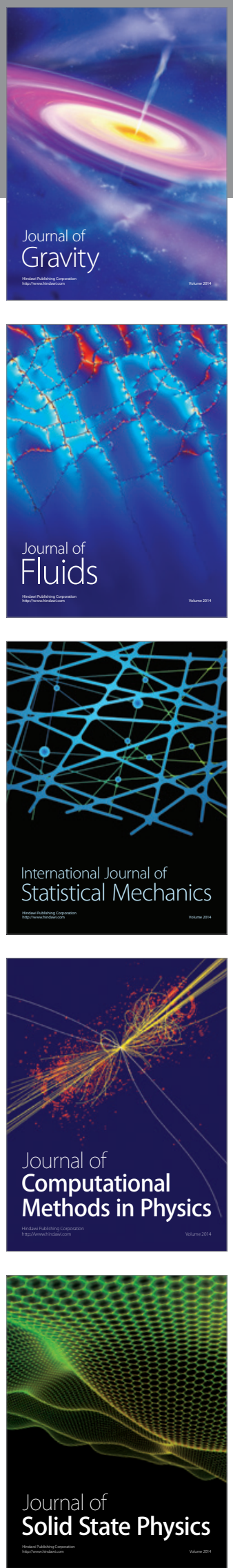

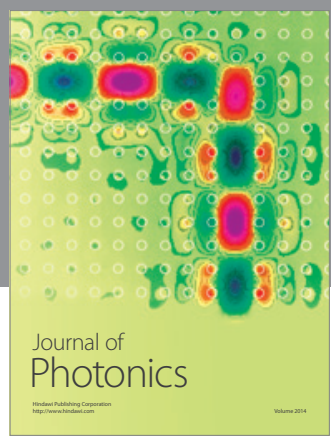

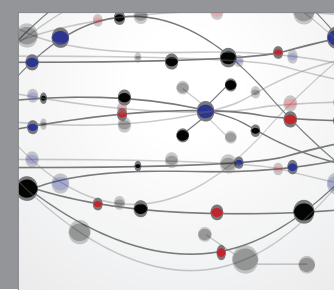

The Scientific World Journal

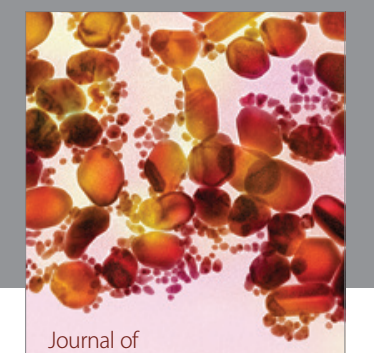

Soft Matter
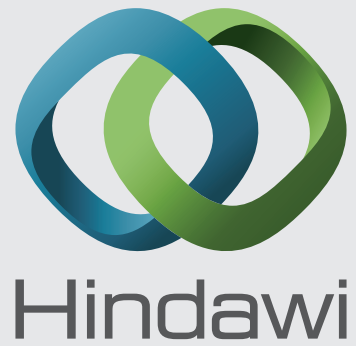

Submit your manuscripts at

http://www.hindawi.com
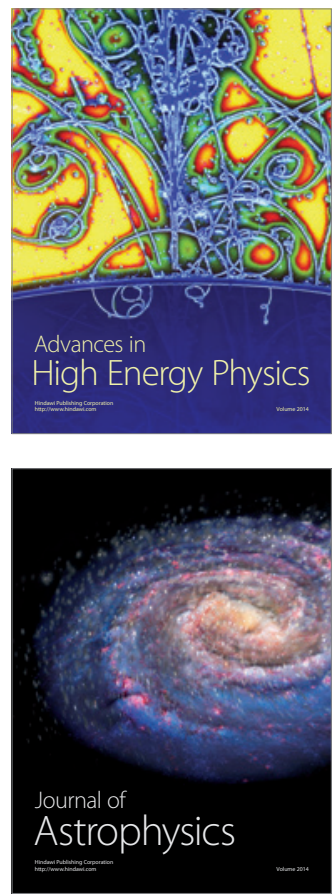
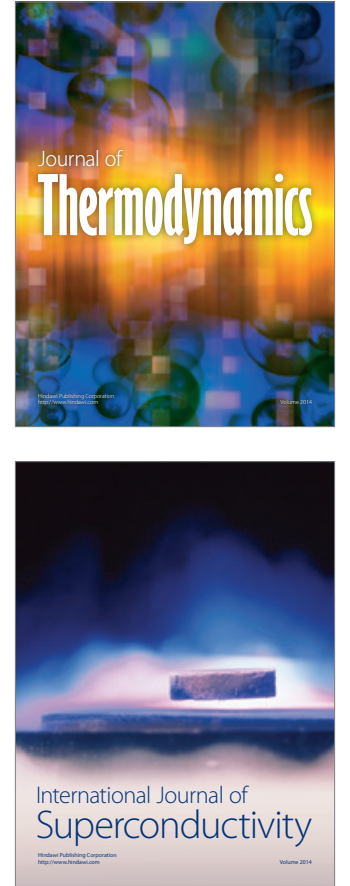
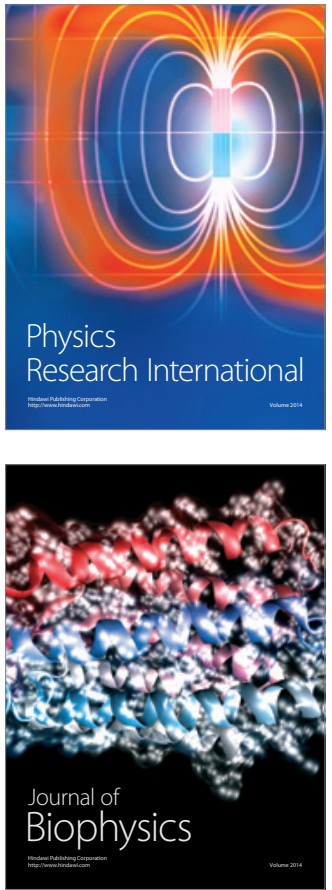
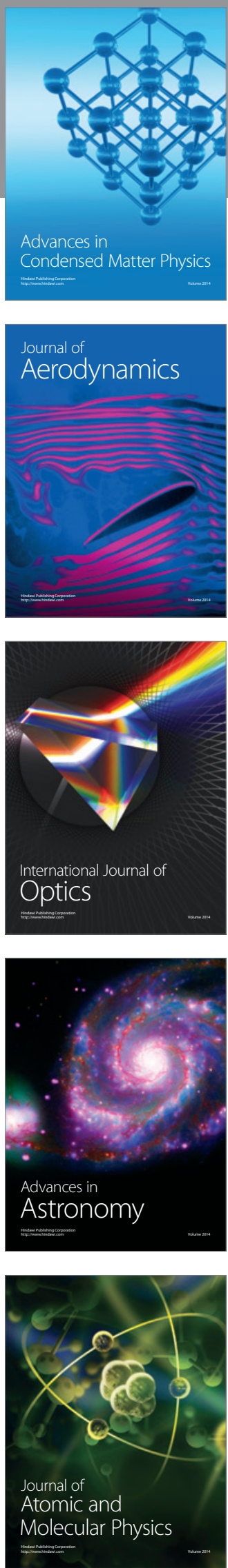